\title{
Autoimmune screening before adenovirus vector-based DNA vaccine in women may avoid underuse for all the subjects
}

\author{
Maria Giulia Mosconi ${ }^{1}$ (D) Francesco Caso ${ }^{2} \cdot$ Giorgio Maraziti $^{1} \cdot$ Christine Kremer $^{3,4} \cdot$ Luisa Costa $^{2} \cdot$ Raffaele Scarpa $^{2}$. \\ Roberto Giacomelli ${ }^{5}$. Valeria Caso ${ }^{1}$
}

Published online: 5 October 2021

(c) Fondazione Società Italiana di Neurologia 2021

Dear Editor,

Initially, the UK COVID-19 distribution strategy for the viral vector vaccine, ChAdOx1 nCoV-19 (AZD1222), was to deliver a single dose to the entire population.

However, March 2021 data from the Medicines and Healthcare Products Regulatory Agency (MHRA) reported 79 cases of immune thrombotic thrombocytopenia (ITT) following the administration of the vaccine: 44 cerebral venous sinus thromboses (CVST), and 35 severe vein thromboses at other sites. All 79 cases had occurred after receiving a first dose of the vaccine, and 51 of these were women aged between 18 and 79 years [1]. The number of vaccinated women with the AZD1222 vaccine was 3525 on a total of 5807 vaccinated people (60.7\%). Regarding mortality, 19 of the 79 people died of which 13 were women. Eleven out of the 19 people who died were under 50 years of age, and 3 were under 30 years of age. Fourteen of these 19 cases were CVST and 5 thromboses with ITT $[1,2]$.

Maria Giulia Mosconi and Francesco Caso contributed equally to this work.

Maria Giulia Mosconi

mariagiulia98581@yahoo.it

1 Stroke Unit and Division of Internal and Cardiovascular Medicine, Santa Maria della Misericordia Hospital, University of Perugia, Piazzale Menghini 1, 06129 Perugia, Italy

2 Department of Clinical Medicine and Surgery, School of Medicine and Surgery, University of Naples Federico II, Naples, Italy

3 Neurology Department, Skåne University Hospital, Malmö, Sweden

4 Department of Clinical Sciences, Lund University, Lund, Sweden

5 Rheumatology and Immunology Unit, Department of Medicine, University of Rome Campus Biomedico, 00128 Rome, Italy
Whereas of March 31 2021, European Union countries had administered a total of 33,385,052 vaccinations, including 5,181,905 women under 50 years of age. The reported incidence of CVST with ITT was 44/5,181,905 (8.5 cases/ million) and the reported overall incidence of venous thrombotic events was 79/5,181,905 (15 cases/million people) [2].

In line with previous research reporting sex differences in vaccine responses, women exhibited a more significant immune response that might facilitate vaccine efficacy, but they also experienced more frequent and more severe adverse events (AEs) [3-5].

The interim analysis of phase $1 / 2 / 3$ ongoing AZD1222 vaccine trials has reported that almost $80 \%$ of the enrolled women were aged between 18 and 55. As of 5807, there were eighty-four (1.45\%) serious AEs reported, but only 2 were considered possibly related to vaccination, a transverse myelitis case and fever, but no specific sex-related AEs were reported [2]. In February 2021, the "Centers for Disease Control and Prevention" (CDC) released data on AEs pertaining to the first month of the COVID-19 vaccine rollout, reporting that women received $61 \%$ of the first vaccine doses, whereas $72 \%$ of the reported side effects occurred in women [6]. These findings suggest that this higher reactivity of the women immune system should be taken into account.

The extremely rare number of cases of ITT following the administration of a single dose of the COVID-19 viral vector vaccine shares many clinical and pathogenetic aspects with another immune-mediated complication affecting a low proportion ( 0.3 to $4.8 \%$ ) of patients with previous heparin exposure, the so-called autoimmune heparin-induced thrombocytopenia (HIT) [7, 8].

The ITTs of vaccinated patients have had a relatively high incidence of venous thrombosis in unusual sites, such as the cerebral veins. This is in line with data from an 11-yearobservational study focusing on HIT patients after highdose heparin administration. In fact, out of the 120 consecutive patients enrolled with thrombotic complications, 
7 suffered from ischemic cerebrovascular events, 3 from cerebral venous thrombosis, and 1 had a transient confusional state. The overall relative mortality was reported to be much higher in HIT patients with neurologic complications (55\%), compared to those patients without neurologic damage (11\%) [9].

Both for HIT and adenovirus vector-based DNA vaccines ITT, a key role is played by the platelet-activating antibodies known as anti-PF4-polyanion complexes. Anti-PF4antibodies bind and activate cellular FcyRIIA expressed on platelets and monocytes, leading to a hypercoagulable state and venous thrombotic events $[10,11]$.

From literature, several cases of HIT in the absence of heparin exposure have been reported, probably triggered by the presence of other polyanions deriving from other molecules, such as chondroitin sulfate, polyphosphates, nucleic acid, bacterial components. Thus, the possible role of viral components, vaccines adjuvants, and/or excipients cannot be excluded [12].

Regarding the ITT case series described by Greinacher et al. [10], in fact, out of the 11 patients, 2 (18.2\%) presented anticardiolipin antibodies, which are typical of Antiphospholipid syndrome (APS). Of note, adenovirus-vector-based DNA-vaccines-ITT also can show several clinical manifestations of severe forms of APS, termed catastrophic APS (CAPS), which can be associated with thrombocytopenia $[10,13]$.

Some studies have reported that PF4 appeared as the dominant $\beta 2$ GPI binding protein and that the interaction anti- $\beta 2$ GPI- $\beta 2$ GPI-PF 4 could probably have been involved in the prothrombotic tendency of APS. In particular, it has been reported that the reactivity in APS was higher against PF4- $\beta 2$ GPI complex than against $\beta 2$ GPI alone. Also, anti$\beta 2$ GPI- $\beta 2$ GPI-PF 4 has been shown thrombo-inflammatory mechanisms as well as the ability to significantly induce platelet p38MAPK phosphorylation and TXB2 production, mainly through $\mathrm{F}\left(\mathrm{ab}^{\prime}\right) 2$ fragments of antibodies [14].

Moreover, due to peculiar sex and hormonal characteristics [15-17], autoimmunity patterns are frequent in young women.

In light of the aforementioned, an autoimmune screening may appropriately be considered on young women before administering a viral vector vaccine, in order to reduce the incidence of the ITT and avoid the vaccine underutilization.

Author contribution MGM conceptualized and supervised the manuscript; wrote, edited, and finalized the manuscript.

FC conceptualized and supervised the manuscript; wrote, edited, and finalized the manuscript-

GM conceptualized and supervised the manuscript; wrote, edited, and finalized the manuscript.

CK wrote, edited, and finalized the manuscript.
LC wrote, edited, and finalized the manuscript.

RS wrote, edited, and finalized the manuscript.

RG wrote, edited, and finalized the manuscript.

VC conceptualized and supervised the manuscript; wrote, edited, and finalized the manuscript.

Data availability N/A.

Code availability N/A.

\section{Declarations}

Conflict of Interest The authors declare no competing interests.

Ethical approval None

\section{References}

1. Medicines and healthcare products regulatory agency. GOV.UK. https://www.gov.uk/government/news/mhra-issues-new-adviceconcluding-a-possible-link-between-covid-19-vaccine-astra zeneca-and-extremely-rare-unlikely-to-occur-blood-clots?utm source $=$ Communications $\% 2$ C $\% 20$ Policy $\% 20$ and $\% 20$ Research \&utm_medium $=$ email\&utm_campaign $=12303649 \_$President $\%$ 27 s $\% 20$ bulletin $\% 20 \% 28$ special $\% 20$ ed $\% 29 \% 20-\% 2012 \% 20$ April $\%$ 202021\&utm_content=HIT-like\%20events\&dm_i=1V12,7BPK1 ,JEDW62,TPWCQ,0

2. Voysey M, Clemens SAC, Madhi SA, et al; Oxford COVID Vaccine Trial Group (2021) Safety and efficacy of the ChAdOx1 nCoV-19 vaccine (AZD1222) against SARS-CoV-2: an interim analysis of four randomised controlled trials in Brazil, South Africa, and the UK. Lancet. 397(10269):99-111. Epub 2020 Dec 8. Erratum in: Lancet. 2021 Jan 9;397(10269):98. https://doi.org/ 10.1016/S0140-6736(20)32661-1

3. Fink AL, Klein SL (2015) Sex and gender impact immune responses to vaccines among the elderly. Physiology (Bethesda) 30(6):408-416. https://doi.org/10.1152/physiol.00035.2015

4. Fischinger S, Boudreau CM, Butler AL, Streeck H, Alter G (2019) Sex differences in vaccine-induced humoral immunity. Semin Immunopathol. 41(2):239-249. Epub 2018 Dec 13. https://doi. org/10.1007/s00281-018-0726-5

5. Flanagan KL (2017) Plebanski M (2016) Sex-differential heterologous (non-specific) effects of vaccines: an emerging public health issue that needs to be understood and exploited. Expert Rev Vaccines 16(1):5-13. https://doi.org/10.1080/14760584.2016.12032 60

6. McCartney PR (2020) Sex-based vaccine response in the context of COVID-19. J Obstet Gynecol Neonatal Nurs 49(5):405-408. https://doi.org/10.1016/j.jogn.2020.08.001

7. Warkentin TE, Sheppard JA, Sigouin CS, Kohlmann T, Eichler P, Greinacher A (2006) Gender imbalance and risk factor interactions in heparin-induced thrombocytopenia. Blood 108(9):29372941. https://doi.org/10.1182/blood-2005-11-012450

8. Cuker A, Arepally GM, Chong BH, Cines DB, Greinacher A, Gruel Y, Linkins LA, Rodner SB, Selleng S, Warkentin TE, Wex A, Mustafa RA, Morgan RL, Santesso N (2018) American Society of Hematology 2018 guidelines for management of venous thromboembolism: heparin-induced thrombocytopenia. Blood Adv 27(22):3360-3392. https://doi.org/10.1182/bloodadvances. 20180244890

9. Pohl C, Harbrecht U, Greinacher A, Theuerkauf I, Biniek R, Hanfland P, Klockgether T (2000) Neurologic complications in 
immune-mediated heparin-induced thrombocytopenia. Neurology 54(6):1240-1245. https://doi.org/10.1212/wnl.54.6.1240

10. Greinacher A, Thiele T, Warkentin TE, Weisser K, Kyrle PA, Eichinger S (2021) Thrombotic thrombocytopenia after ChAdOx1 nCov-19 vaccination. N Engl J Med 384(22):2092-2101. https:// doi.org/10.1056/NEJMoa2104840

11. Arepally GM (2017) Heparin-induced thrombocytopenia. Blood 129(21):2864-2872. https://doi.org/10.1182/ blood-2016-11-709873

12. Marchetti M, Zermatten MG, Bertaggia Calderara D, Aliotta A, Alberio L (2021) Heparin-induced thrombocytopenia: a review of new concepts in pathogenesis, diagnosis, and management. J Clin Med 10(4):683. https://doi.org/10.3390/jcm10040683

13. Papadakis E, Banti A, Kioumi A (2016) Women's issues in antiphospholipid syndrome. Isr Med Assoc J. 18(9):524-529

14. Sikara MP, Routsias JG, Samiotaki M, Panayotou G, Moutsopoulos HM, Vlachoyiannopoulos PG (2010) \{beta\}2 Glycoprotein I (\{beta\}2GPI) binds platelet factor 4 (PF4): implications for the pathogenesis of antiphospholipid syndrome. Blood 115(3):713723. https://doi.org/10.1182/blood-2009-03-206367

15. Chaturvedi S, Braunstein EM, Brodsky RA (2021) Antiphospholipid syndrome: complement activation, complement gene mutations, and therapeutic implications. J Thromb Haemost 19(3):607616. https://doi.org/10.1111/jth.15082

16. Papadakis E, Banti A, Kioumi A (2016) Women's issues in antiphospholipid syndrome. Isr Med Assoc J 18(9):524-529

17. Angum F, Khan T, Kaler J, Siddiqui L, Hussain A (2020) The Prevalence of Autoimmune Disorders in Women: A Narrative Review. Cureus 12(5):e8094. https://doi.org/10.7759/cureus.8094

Publisher's note Springer Nature remains neutral with regard to jurisdictional claims in published maps and institutional affiliations. 